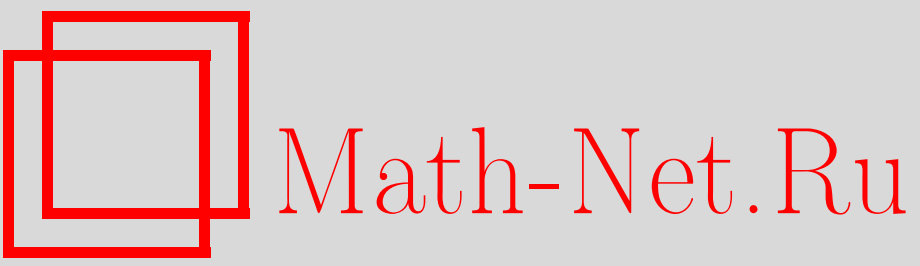

Н. М. Адрианов, Г. Б. Шабат, Функции Белого 4-реберных детских рисунков рода 2, УМН, 2005, том 60, выпуск 6, 229-230

DOI: https://doi.org/10.4213/rm1686

Использование Общероссийского математического портала Math-Net.Ru подразумевает, что вы прочитали и согласны с пользовательским соглашением

http://www.mathnet.ru/rus/agreement

Параметры загрузки:

IP: 52.90.164.192

26 апреля 2023 г., 14:29:07 


\title{
ФУНКЦИИ БЕЛОГО 4-РЕБЕРНЫХ ДЕТСКИХ РИСУНКОВ РОДА 2
}

\author{
Н. М. АДРИАНОВ, Г. Б. ШАБАТ
}

1. Введение. Детским рисунком мы назьваем графф Г, вложенный в связную компактную ориентированную поверхность $\mathscr{X}$ таким образом, что дополнение $\mathscr{X} \backslash \Gamma$ гомеоморфоно несвязному объединению открытых дисков. Пусть $X$ - полная гладкая неприводимая комплексная кривая. Функция $\beta$ на $X$ называется (чистой) функцией Белого, если $\beta$ не имеет критических значений кроме 0,1 и $\infty$, причем все прообразы $\beta^{-1}(1)$ двукратны. Пара $(X, \beta)$, где $\beta$ - функция Белого, называется парой Белого.

Пусть $(X, \beta)$ - пара Белого. Рассмотрим граф $\Gamma=\beta^{-1}([0,1])$ на $X$, вершинами которого являются прообразы $\beta^{-1}(0)$. Тогда $(X, \Gamma)$ - детский рисунок, причем каждый детский рисунок можно задать единственной (с точностью до изоморфизма) парой Белого.

Термин “детский рисунок” был предложен А. Гротендиком [1], который и обратил внимание на связь графов на поверхностях с алгебраическими кривьми и теорией Галуа. Современный обзор теории детских рисунков можно найти в [2].

2. Перечисление. Поскольку у рисунка есть, как минимум, одна вершина и одна грань, из формулы Эйлера $V-E+F=2-2 g$ видно, что для рода $g=2$ минимально возможное количество ребер равно 4. Несложно перечислить 4-реберные детские рисунки рода 2. Таких рисунков существует всего 4, соответствующие склейки 8-угольника задаются гауссовыми словами $a b c d a b c d, a b a b c d c d, a b a c b d c d, a b a c d b c d$ и изображены на рис. 1.

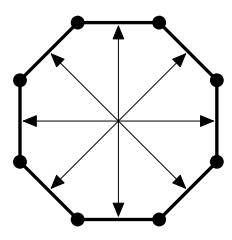

$A$

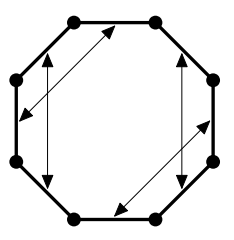

$B$

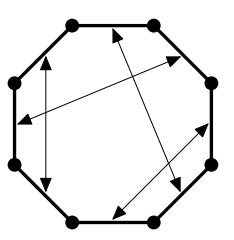

$C_{1}$

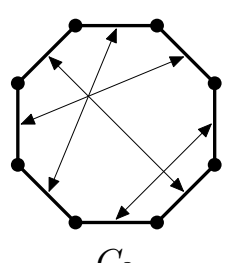

$C_{2}$

Рис. 1. Склейки 8-угольника рода 2

Группа автоморфизмов $\operatorname{Aut}(D)$ одноклеточного рисунка $D$, представленного в виде склейки правильного многоугольника, есть циклическая группа, состоящая из вращений многоугольника, оставляющих склейку неизменной. Легко видеть, что $\operatorname{Aut}(A)$ - циклическая группа порядка $8, \operatorname{Aut}(B)$ - циклическая группа порядка $2, \operatorname{Aut}\left(C_{1}\right)$ и $\operatorname{Aut}\left(C_{2}\right)$ - тривиальны.

3. Самодвойственность. Если пара Белого $(X, \beta)$ задает детский рисунок $D$, то пара $(X, 1 / \beta)$ также является парой Белого и задает двойственньй рисунок $D^{*}$. Вершины рисунка $D^{*}$ совпадают с центрами граней рисунка $D$, и наоборот. Рисунок $D$ называется самодвойственным, если он изоморфен двойственному: $D \simeq D^{*}$. В терминах пары Белого $(X, \beta)$ это означает, что существует автоморфизм кривой $\tau: X \rightarrow X$ такой, что $\tau^{*} \beta=1 / \beta$.

Поскольку группы автоморфизмов рисунков $D$ и $D^{*}$ изоморфны, рисунки $A$ и $B$ самодвойственны: они однозначно определяются родом, количеством ребер и группой автоморфизмов. Случай рисунков $C_{1}$ и $C_{2}$ требует более тщательных рассмотрений: они могли бы быть двойственны друг другу. Тем не менее, оказывается верньм следующее предложение.

ПреДЛОЖениЕ. Рисунки $A, B, C_{1}, C_{2}$ - самодвойственные.

4. Пары Белого. Пусть $(X, \beta)$ - пара Белого одного из рисунков $A, B, C_{1}, C_{2}$. Тогда дивизор функции Белого имеет вид $(\beta)=8\left(A_{0}-A_{\infty}\right)$, где $A_{0}, A_{\infty} \in X$ - единственный нуль и единственный полюс функции $\beta$.

Пусть $\mathrm{Cl}(X)$ - группа классов дивизоров алгебраической кривой $X$, см. [3]. Будем обозначать $m \Gamma$ подгруппу $m$-кручения абелевой группы $\Gamma$. Тогда из условия $(\beta)=8\left(A_{0}-A_{\infty}\right)$ следует, что $A_{0}-A_{\infty} \in{ }_{8} \mathrm{Cl}(X)$ и имеется 3 возможности:
(A) $A_{0}-A_{\infty} \in{ }_{2} \mathrm{Cl}(X)$
(B) $A_{0}-A_{\infty} \in{ }_{4} \mathrm{Cl}(X) \backslash{ }_{2} \mathrm{Cl}(X)$,
(C) $A_{0}-A_{\infty} \in{ }_{8} \mathrm{Cl}(X) \backslash_{4} \mathrm{Cl}(X)$. 
Оказывается, что все они реализуются: случай $(A)$ соответствует рисунку $A$, случай $(B)$ - рисунку $B$, случай $(C)$ - рисункам $C_{1}$ и $C_{2}$. В случае $(A)$ функция $\beta$ является четвертой степенью некоторой функции на $X$, в случае $(B)$ - квадратом некоторой функции на $X$. В случае $(C)$ дивизор $4\left(A_{0}-A_{\infty}\right)$ имеет порядок 2 в $\mathrm{Cl}(X)$, т.е. он линейно эквивалентен дивизору $W_{1}-W_{2}$, где $W_{1}, W_{2}$ - точки Вейерштрасса гиперэллиптической кривой $X$. Следовательно, в этом случае $\beta$ можно представить в виде

$$
\beta=\frac{x-x\left(W_{1}\right)}{x-x\left(W_{2}\right)} h^{2}=\frac{\left(\left(x-x\left(W_{1}\right)\right) h\right)^{2}}{\left(x-x\left(W_{1}\right)\right)\left(x-x\left(W_{2}\right)\right)} .
$$

Описать все дальнейшие детали вычислений не позволяет объем заметки, поэтому ограничимся формулировкой результата.

Tеорема. (i) Пара Белого рисунка $А$ задается уравнениями

$$
y^{2}=x^{5}-x, \quad \beta=x^{4} .
$$

(ii) Пара Белого рисунка В задается уравнениями

$$
y^{2}=\left(x^{2}-2\right)\left(x^{4}-2 x^{2}+2\right), \quad \beta=-\left((1-x)^{2}(1+x)^{2}-x y\right)^{2} .
$$

(iii) Положим $\varepsilon=1 \pm \sqrt{2}, \omega=\varepsilon+1 / \varepsilon+1=1 \pm 2 \sqrt{2}$,

$$
\begin{aligned}
& Q_{2}(x)=(x-\varepsilon)(x-1 / \varepsilon)=x^{2}+(1-\omega) x+1, \\
& Q_{3}(x)=(x+1)\left(x^{2}-2 \varepsilon x+1\right), \\
& E_{2}(x)=-\omega x^{2}+6 x-\omega, \\
& E_{4}(x)=\left(x^{2}-2 \varepsilon x-\omega\right)\left(-\omega x^{2}-2 \varepsilon x+1\right) .
\end{aligned}
$$

Тогда пары Белого рисунков $C_{1}$ и $C_{2}$ задаются уравнениями

$$
y^{2}=E_{2}(x) E_{4}(x), \quad \beta=-\frac{1}{16 \varepsilon^{2}} \frac{\left(Q_{3}(x) E_{2}(x)+Q_{2}(x) y\right)^{2}}{E_{2}(x)} .
$$

Значение $\varepsilon=1-\sqrt{2}$ соответствует рисунку $C_{1}$, а значение $\varepsilon=1+\sqrt{2}-$ рисунку $C_{2}$.

\section{СПИСОК ЛИТЕРАТУРЫ}

[1] A. Grothendieck // London Math. Soc. Lecture Notes Series. 1997. V. 242. P. 5-48. [2] S. K. Lando, A. K. Zvonkin. Graphs on Surfaces and Their Applications. Berlin: SpringerVerlag, 2004. (Encyclopedia Math. Sci. V. 141.) [3] Р. Хартсхорн. Алгебраическая геометрия. М.: Мир, 1981.

Московский государственный университет им. М.В.Ломоносова; Российский государственный гуманитарньй университет, Независимьй московский университет

E-mail: nikolai_adrianov@cnit.msu.ru; george@shabat.mccme.ru 